\title{
COMBINATORIAL AUCTIONS FOR SELLING NETWORK CAPACITY
}

The network economy is a term for today's global relationship among economic subjects characterized by massive connectivity. Today's network systems provide the infrastructure and foundation for the functioning of societies and economies. They come in many forms and include physical networks such as transportation and logistical networks, communication networks, energy networks, as well as more abstract networks as economic, financial, social, and knowledge networks. The paper presents modeling of auctions in network economy. Auctions are important market mechanisms for the allocation of goods and services. Combinatorial auctions are those auctions in which bidders can place bids on combinations of items. Combinatorial auction is an appropriate instrument for selling network capacity. The winner determination problem in a combinatorial auction for selling network capacity is formulated. The model can be formulated as a multicommodity flow problem and efficient algorithms can be used.

Keywords: Network economy, combinatorial auction, winner determination problem, multicommodity network flows

\section{Introduction}

The paper presents modeling of combinatorial auctions in network economy. It is an integration of two characteristic elements of today's economic reality, the network economy and auction mechanisms. The network economy is a term for today's global relationship among economic subjects characterized by massive connectivity. Network industries play a crucial role in modern life. Today's network systems provide the infrastructure and foundation for the functioning of societies and economies. Auctions are important market mechanisms for the allocation of goods and services. Auctions are preferred often to other common processes because they are open, quite fair, easy to understand by participants, and lead to economically efficient outcomes. Their popularity is also caused by expansion of e-commerce. Design of auctions (see [7]) is a multidisciplinary effort made of contributions from economics, operations research, computer science, and other disciplines.

There is a possible classification of auctions by different aspects:

1. Traded items (indivisible, divisible, pure commodities, structured commodities).

2. Participants' roles in auctions (one-sided, multilateral auctions).

3. Objectives of auctions (optimization, allocation rules, pricing rules).

4. Complexity of bids (simply, related bids).

5. Organization of auctions (single-round, multi-round, sequential, parallel, price schemes).

For auctions for selling network capacity it is useful to use so called combinatorial auctions. Combinatorial auctions refer to auctions in which participants are allowed to bid on combinations of items. A classical problem of combinatorial auctions is the winner determination problem. The problem can be formulated as an integer programming problem and is well-known to be NPhard. In the paper the winner determination problem for selling network capacities is formulated. The model is based on combinatorial auctions with a network structure of items.

\section{Network economy}

The network economy (see [4], [5], [9]) is a term for today's global relationship among economic subjects characterized by massive connectivity. The central act of the new era is to connect everything to everything in deep web networks at many levels of mutually interdependent relations, where resources and activities are shared, markets are enlarged and costs of risk are reduced. Connections are enabled by an explosive development of information and communication technologies. Network connections enable tighter relations between firms and stakeholders. New technologies provide a permanent feedback that enables activity modifications and quick responses and therefore fundamentally change business models. Network industries play a crucial role in modern life. Today's network systems provide the infrastructure and foundation for the functioning of societies and economies. They come in many forms and include physical networks such as: transportation and logistical networks, communication networks, energy networks, as well as more abstract networks comprising: economic and financial networks, environmental networks, social, and knowledge networks. Many important non-network industries share many essential economic features with network industries. These nonnetwork industries are characterized by strong complementary relations.

\footnotetext{
* Petr Fiala

Department of Econometrics, Faculty of Informatics and Statistics, University of Economics, W. Churchill Sq. 4, 13067 Prague 3, E-mail: pfiala@vse.cz
} 
The reality of today's networks includes features:

- large-scale nature and complexity,

- increasing congestion,

- complementarity,

- externalities,

- switching costs,

- alternative behaviors of users of the networks,

- interactions between the networks themselves.

Many of today's networks are characterized by both a largescale nature and complexity of the network topology. Congestion is playing an increasing role in not only transportation networks but also in telecommunication networks. The crucial relationship in networks is the complementarity between the pieces of the network. Complementarity turns out to be a crucial factor in the markets for information goods. Networks exhibit positive externalities. The value of a unit increases with the expected number of units to be sold. Costs of switching to a different service or adopting a new technology are significant. The decisions made by the users of the networks, in turn, affect not only the users themselves but others, as well, in terms of profits and costs, timeliness of deliveries, the quality of the environment, etc. The behavior of the users of the networks themselves may be non-cooperative. An example is behavior of users of transport or telecommunication networks, where optimization from single users' perspective may not be optimal from a system one. This situation is illustrated by the famous Braess's paradox (see [2]), where an addition of a new link with identical demand faces the increasing costs for all users.

\section{Example 1}

Braess's paradox is illustrated by a simple example. The original network (Fig. 1) consists of four nodes 1, 2, 3, 4 and four edges $h_{1}, h_{2}, h_{3}, h_{4}$, the origin and the destination of the network are presented by nodes 1 and 4 .

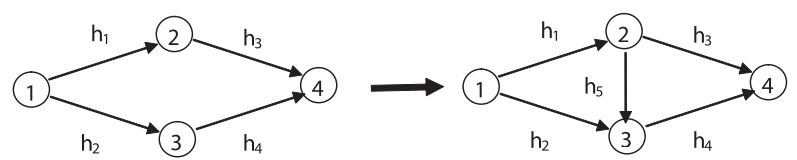

Fig. 1 Braess's paradox

There are two paths from the origin to the destination of the network $C_{1}=\left\{h_{1}, h_{3}\right\}$ a $C_{2}=\left\{h_{2}, h_{4}\right\}$. Let we suppose the costs on the edges depending on the flow quantities $x_{1}, \ldots, x_{4}$ are

$$
\begin{aligned}
& n_{1}\left(x_{1}\right)=10 x_{1}, n_{2}\left(x_{2}\right)=x_{2}+50, n_{3}\left(x_{3}\right)=x_{3}+50, \\
& n_{4}\left(x_{4}\right)=10 x_{4}
\end{aligned}
$$

and the total required network flow $X=6$.

In the case of user-optimization, the equilibrium solution is given by the situation, where all paths connecting the origin-destination pair have equal and minimal costs and therefore no user has any incentive to switch this path. The equilibrium solution is given by flows on edges

$$
x_{1}^{*}=3, x_{2}^{*}=3, x_{3}^{*}=3, x_{4}^{*}=3
$$

and by associated path costs

$$
n\left(C_{1}\right)=83, n\left(C_{2}\right)=83 .
$$

A new edge $h_{5}$ joining node 2 to node 3 with user cost $n_{5}\left(x_{5}\right)=x_{5}+10$ is added. The change creates new path $C_{3}=$ $=\left\{h_{1}, h_{5}, h_{4}\right\}$. The original solution is no longer in equilibrium. The new equilibrium solution has the flow of amount 2 for all three paths. Edge flows are

$$
x_{1}^{*}=4, x_{2}^{*}=2, x_{3}^{*}=2, x_{4}^{*}=4, x_{5}^{*}=2
$$

and the associated path costs are

$$
n\left(C_{1}\right)=92, n\left(C_{2}\right)=92, n\left(C_{3}\right)=92 .
$$

Costs grow up for every user of the network from the value 83 to the value 92 . This cost increase is caused by the fact that edges $h_{1}$ and $h_{4}$ are shared by two paths and the flows and cost are increasing on these edges. The addition of a path connecting an origin-destination pair that shares no links with the original connection will never result in Braess's paradox.

\section{Combinatorial auctions}

An auction mechanism is denoted as a combinatorial auction, if combinations of items are traded and not single items only (see [3], [8]). Combinatorial auctions are increasingly considered as an alternative to simultaneous single-item auctions. The advantage of combinatorial auctions is the more precise expression of bidder's preferences. This advantage is primarily important in the case of complementary items. The items are complementary, if the utility of set of items is greater than a sum of utilities of single items. Two items A and B are complementary, if it holds

$$
u(\{A, B])>u([A\})+u(\{B\}) .
$$

From different types of combinatorial auctions we present an auction of indivisible items with one seller and several buyers. Let us suppose that one seller offers a set $G$ of $m$ items, $j=1,2, \ldots, m$, to $\mathrm{n}$ potential buyers. Items are available in single units. A bid made by buyer $i, i=1,2, \ldots, n$, is defined as

$$
N_{i}=\left\{S, p_{i, S}\right\}
$$

where

$S \square G$, is a combination of items,

$p_{i, S} \quad$ is the offered price by buyer i for the combination of items $S$.

The objective is to maximize the revenue of the seller given the bids made by buyers. Constraints establish that no single item is allocated to more than one buyer and that no buyer obtains more than one combination. The winner determination problem belongs to NP-hard problems. 
Notations and variables are introduced for model formulation: $\delta_{j, S}=1$, if item $j \square S, \delta_{j, S}=0$, if item $j \square S$,

$x_{i, S}$ is a bivalent variable specifying if the combination $S$ is assigned to buyer $i\left(x_{i, S}=1\right)$.

The winner determination problem can be formulated as follows

$$
\sum_{i=1}^{n} \sum_{S \subseteq G} p_{i, S} x_{i, S} \rightarrow \max
$$

subject to

$$
\begin{aligned}
& \sum_{i=1}^{n} \sum_{S \subseteq G} \delta_{j, S} x_{i, S} \square 1, \forall j \square G, \\
& \sum_{S \subseteq G} x_{i, S} \square 1, \forall i, i=1,2, \ldots, n, \\
& x_{i, S} \square\{0,1\}, \forall S \square G, \forall i, i=1,2, \ldots, n .
\end{aligned}
$$

For general solving of the winner determination problem, dynamic programming (see [6]) is proposed. The authors also consider several restrictions on allowable bids that make the problem computationally manageable.

\section{Auctions on networks}

The traded commodities can be network capacities, which enable various types of flows in network industries. A classical example is capacity of telecommunication networks where the capacities of certain links are supplied and demanded. The objective is the optimal combination of segments in required paths. Principles of combinatorial auctions are useful for selling network capacities. The utility of the path capacity is greater than a sum of utilities of edge capacities.

For illustration we present a basic formulation of the winner determination problem in a combinatorial auction for selling network capacities. Let $G=(U, H)$ be a network, where $U$ is a set of nodes and $H$ a set of edges. To each edge $h_{j} \square H, j=1,2, \ldots, m$ a capacity $k\left(h_{j}\right)$ is associated. Capacities of the network are owned by a single seller and there are $n$ buyers, potentially interested in path capacities. The combinatorial aspect of the problem ensues from the fact that buyers desire to obtain path capacities (combinations of edges) rather than on individual capacities of edges. We suppose that buyer $i, i=1,2, \ldots, n$, submits a single bid specified by the following specifications

$$
N_{i}=\left\{Z_{i}, K_{i}, G_{i}, k_{i}, p_{i}\right\},
$$

where

$Z_{i}, K_{i}$ is an origin-destination pair of nodes specifying the path for buyer $i$,

$G_{i} \quad$ is a subgraph of the graph $G$ specifying edges for possible paths,

$k_{i} \quad$ is the required capacity for the path, $p_{i} \quad$ is the offered price of buyer $i$ for the combination.

Other notations and variables are introduced for model formulation:

$C_{i}$ is the set of all paths between $Z_{i}$ and $K_{i}$ in the subgraph $G_{i}$, $c$ is a path from the set $C_{i}$,

$\delta\left(h_{j}, c\right)=1$, if $h_{j} \square c, \delta\left(h_{j}, c\right)=0$, if $h_{j} \square c$,

$y_{c}$ is a variable specifying the capacity on the path $c \square C_{i}$,

$x_{i}$ is a bivalent variable specifying if bid $N_{i}$ is winning $\left(x_{i}=1\right)$.

The winner determination problem in a combinatorial auction for selling network capacities can be formulated as follows

$\sum_{i=1}^{n} p_{i} x_{i} \rightarrow \max$

subject to

$$
\begin{aligned}
& \sum_{c \in C_{i}} y_{c}=k_{i} x_{i}, i=1,2, \ldots, n, \\
& \sum_{i=1}^{n} \sum_{c \in C_{i}} \delta\left(h_{j}, c\right) y_{c} \square k\left(h_{j}\right), h_{j} \square H, \\
& x_{i} \square\{0,1\}, i=1,2, \ldots, n, \\
& y_{c} \square 0, c \square C_{i}, i=1,2, \ldots, n .
\end{aligned}
$$

When paths are specified by buyers and single units of capacity are available on edges as requested by buyer, the model is equivalent to the model of the winner determination problem, in which items are edges and combinations are paths. The particularity of the model lies in the fact that buyers do not need to indicate a specific path along which the capacity should be allocated. It is an auctioneer's task of routing the requested capacities in order to determine the winning bids. The model could be solved by commercial mixed integer programming software. However, the model can be formulated as a multicommodity flow problem. Multicommodity network flow problems involve several flow types or commodities, which simultaneously use the network and are coupled through edges with limited capacity. Each commodity has an associated demand and an origin-destination pair of nodes. Important examples of such problems arise in communication, transportation, and manufacturing networks. Effective methods can be used for solving multicommodity network problems (see [1]). The solution methods for solving the multicommodity flow problem generally attempt to exploit the network structure of the individual commodity flow problems. The approaches are based on pricedirective decomposition, resource-directive decomposition, or partitioning methods.

\section{Example 2}

This simple example is an illustration of terms and notations. Fig. 2 presents a network with following capacities

$$
\begin{aligned}
& k\left(h_{1}\right)=3, k\left(h_{2}\right)=3, k\left(h_{3}\right)=3, k\left(h_{4}\right)=3, k\left(h_{5}\right)=3, \\
& k\left(h_{6}\right)=3, k\left(h_{7}\right)=3, k\left(h_{8}\right)=3, k\left(h_{9}\right)=3 .
\end{aligned}
$$




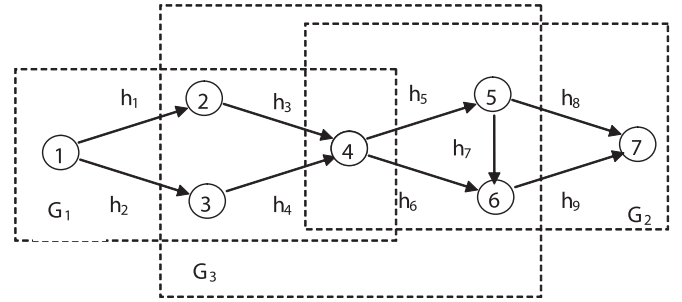

Fig. 2 Selling network capacities

Let us suppose three bidders with their bids

$$
\begin{aligned}
& N_{1}=\left\{Z_{1}=1, K_{1}=4, G_{1}, k_{1}=3, p_{1}=10\right\}, \\
& N_{2}=\left\{Z_{2}=4, K_{2}=7, G_{2}, k_{2}=3, p_{1}=10\right\}, \\
& N_{3}=\left\{Z_{3}=3, K_{3}=6, G_{3}, k_{3}=3, p_{3}=10\right\} .
\end{aligned}
$$

Subgraphs $G_{1}, G_{2}, G_{3}$ specifying edges for possible paths are shown in Fig. 2. However, for the bid $N_{3}$ the edge $h_{3}$ will be never used.

There is the optimal solution of the example

$x_{1}=1, x_{2}=1, x_{3}=1, \sum_{i=1}^{n} p_{i} x_{i}=30$.

The paths are identified for particular bids

$C_{1}=\left\{h_{1}, h_{3}\right\}$,

$$
\begin{aligned}
& C_{2}=\left\{h_{6}, h_{9}\right\}, \\
& C_{3}=\left\{h_{4}, h_{5}, h_{7}\right\}
\end{aligned}
$$

or alternatively

$$
\begin{aligned}
& C_{1}=\left\{h_{1}, h_{3}\right], \\
& C_{2}=\left\{h_{5}, h_{8}\right], \\
& C_{3}=\left\{h_{4}, h_{6}\right\} .
\end{aligned}
$$

\section{Conclusions}

Analysis and optimization of network economy functioning are challenges for application of modeling approaches. Auctions are important market mechanisms for the allocation of goods and services. Recently research and applications of combinatorial auctions are significantly increasing. In the paper a basic model for selling network capacity is formulated. The model is based on combinatorial auctions. The advantage for solving this model is a possibility of utilization algorithms for multicommodity network problems. This basic model can be extended for other types of auctions on network structures. Integration of findings from economics, operations research and computer science is promising for interesting results.

The research project was supported by Grant No. 402/05/0148 from the Grant Agency of the Czech Republic "Network economy - modeling and analysis.”

\section{References}

[1] AHUJA, R. K., MAGNANTI, T. L., ORLIN, J. B. (1993): Network flows: Theory, algorithms, and applications. Prentice Hall, Englewood Cliffs.

[2] BRAESS, D. (1968): Über ein Paradoxon aus der Verkehrsplanung. Unternehmensforschung 12, 258-268.

[3] CRAMTON, P., SHOHAM, Y., STEINBERG, R. (eds.) (2006): Combinatorial Auctions. MIT Press, Cambridge, Massachusetts.

[4] ECONOMIDES, N. (1996): The Economics of Networks. International Journal of Industrial Organization 14, no. 6, 673-699.

[5] FIALA P. (2005): Network's economics. Automa 11, no. 7, 13-15.

[6] PEKEČ, A., ROTHKOPF, M. H. (2003): Combinatorial auction design. Management Science 49, no. 11, 1485-1503.

[7] ROTHKOPF, M. H., PARK, S. (2001): An elementary introduction to auctions. Interfaces 31, no. 6, 83-97.

[8] ROTHKOPF, M. H., PEKEČ, A., HARSTAD, R. M. (1998): Computationally manageable combinatorial auctions. Management Science 44, 1131-1147.

[9] SHAPIRO, C., VARIAN, H. (1999). Information Rules: A Strategic Guide to the Network Economy. Harvard Business School Press, Boston. 